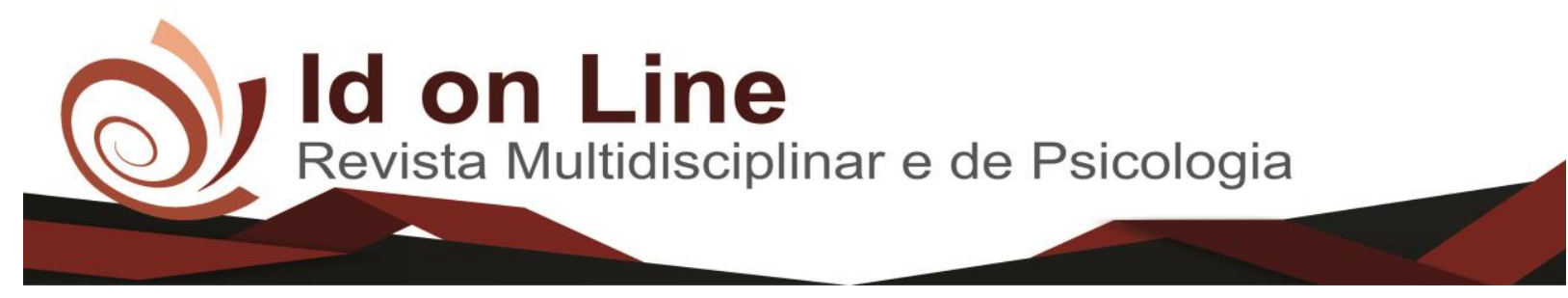

Artigo

\title{
Avaliação das interações medicamentosas potenciais no âmbito da UTI adulta
}

Júlia Souza e Silva ${ }^{l}$; Rodrigo Santos Damascena ${ }^{2}$.

\begin{abstract}
Resumo: As interações medicamentosas potenciais (IMP) tem sido alvo de diversos estudos, que avaliam seus diferentes riscos ou benefícios para o paciente. Daí a importância da identificação e rastreamento de tais efeitos, à partir da identificação das prescrições medicamentosas. O objetivo do presente estudo foi avaliar a frequência de potenciais interações medicamentosas em prescrições médicas nas unidades de terapia intensiva de adulto, do Hospital Público de Vitória da Conquista/BA, em 2017. Os resultados demonstraram um alto índice de efeitos colaterais das interações medicamentosas, suscitando o debate para uma maior atenção nas prescrições, de forma a minimizar-se tal problemática.
\end{abstract}

Palavras-chave: Interações medicamentosas, riscos, efeitos colaterais.

\section{Evaluation of potential drug interactions within the adult ICU}

Abstract: Potential drug interactions (IMP) have been the subject of several studies, which assess their different risks or benefits for the patient. Hence the importance of identifying and tracking these effects, based on the identification of the drug prescriptions. The objective of the present study was to evaluate the frequency of potential drug interactions in medical prescriptions in the adult intensive care units of the Public Hospital of Vitória da Conquista / BA in 2017. The results showed a high index of side effects of drug interactions, raising the debate to increased attention on the requirements, to minimize such is problematic.

Keywords: Drug interactions, risks, side effects.

\section{Introdução}

As interações medicamentosas potenciais (IMP) tem sido alvo de estudos, onde sua ocorrência pode resultar em diferentes riscos ou benefícios para o paciente. Com isso, a identificação e o rastreamento destes efeitos, a partir da identificação das peculiaridades das prescrições medicamentosas, tornam-se de extrema importância. (MAZZOLA; RODRIGUES; DA CRUZ; 2011).

\footnotetext{
${ }^{1}$ Graduação em Farmácia pela Faculdade Independente do Nordeste - FAINOR. Contato: julia.farma28@ gmail.com;

${ }^{2}$ Graduação em Ciências Farmacêuticas pela Universidade Estadual de Feira de Santana. Mestre em Saúde Pública pela Fiocruz. Especialista em Microbiologia Clínica. Especialista em Saúde Pública com Ênfase em PSF. Especialista em Gestão da Assistência Farmacêutica. Especialista em Farmácia Clínica e Hospitalar. Docente do Curso de Especialização em Saúde Pública com Ênfase no NASF da Unigrad Pós-graduação e Extensão.
} 
A partir disso, percebe-se a importância de elucidar acerca da prescrição dos medicamentos, já que isso pode causar diversos tipos de efeitos para o organismo humano.

A interação medicamentosa ocorre quando um fármaco se altera na presença de outro, causando a interrupção de seu efeito clínico ou causando efeitos adversos variados e isso pode diminuir ou elevar os efeitos terapêuticos, podendo ser tóxico ou letal. Nesse sentido, essa interação pode ocorrer também na presença de algum alimento, bebida ou algum agente químico ambiental. (HOEFLER, 2005).

Um dos principais riscos advindos da interação medicamentosa é justamente quando ocorre de os fármacos potencializarem sua ação tóxica, podendo, em casos extremos, causar até a morte. (MAZZOLA; RODRIGUES; DA CRUZ; 2011).

Assim sendo, percebe-se que a interação medicamentosa pode ocorrer com a utilização de dois medicamentos que, ao interagirem, causam diversos efeitos no organismo.

O efeito nocivo que uma interação medicamentosa pode causar no organismo humanoa potencialização do efeito, o corte total deste ou a redução da eficácia de,em alguns casos, com referência a necessidade de uso, pode também levar o paciente a óbito pela ausência da terapêutica do medicamento. (HOEFLER, 2005).

Dentre os casos de interação medicamentosa cabe também destacar as que ocorrem de forma benéfica, pois se tratam de uma interação desejada, de forma prescrita pelo médico ou profissional responsável. (HOEFLER, 2005).

Dessa forma, entende-se que ela também pode ocorrer de maneira a auxiliar em um tratamento medicamentoso, dependendo da dosagem, forma de administração e combinação dos fármacos, e isso, ocorre, impreterivelmente com a orientação médica ou de um profissional competente.

No âmbito hospitalar é de conhecimento geral que o uso de medicação é alto, e que consequentemente pelo uso em massa que existe a grande possibilidade de ocorrerem interações medicamentosas, podendo essas, serem maléficas ou benéficas, e certamente que isso é pautado de acordo, com a orientação racional do uso deste medicamento,

Um estudo realizado dentro da UTI adulto de um Hospital privado de Minas Gerais aponta que, a incidência das interações medicamentosas pode variar de $3 \%$ a $5 \%$ nos pacientes que recebem poucos medicamentos e de até $20 \%$ nos pacientes que recebem de 10 a 20 drogas. Entendendo que a frequência de interações em pacientes em estado critico de saúde pode ser alta, visto o grande número de drogas que recebem. (YUNES et al. 2011). 
Esse estudo evidenciou alta prevalência de interações na UTI, principalmente do tipo moderada, levando-se em conta as condições clínicas desfavoráveis do paciente, alterações fisiológicas e em muitos casos a idade avançada, esse tipo de interação acabam por se tornar mais relevantes. (YUNES et al. 2011).

Portanto, entende-se que em um âmbito geral a interação medicamentosa pode ser um risco a saúde como também um auxilio para a medicina e pacientes.

A partir disso, essa pesquisa se propõe a analisar e identificar as interações medicamentosas observadas em prescrições nas Unidades de Terapia Intensiva (UTIs) adulta de uma unidade hospitalar.

A escolha por identificar as interações medicamentosas que ocorrem dentro de uma Unidade de Terapia Intensiva adulta foi pensada devido ao alto risco que elas podem trazer ao paciente que se encontra em tratamento com fármacos.

Nesse sentido, torna-se importante analisar os índices, para que dessa forma possa haver uma diminuição dos casos e da gravidade desses, bem como, uma interação multiprofissional que colabore com essa perspectiva.

\section{Problema}

Quais as interações medicamentosas potenciais mais frequentes nas unidades de terapia intensiva de adulto do Hospital Público da cidade de Vitória da Conquista/BA no decorrente ano?

\section{Objetivos}

Os objetivos do presente estudo são: - avaliar a frequência de potenciais interações medicamentosas em prescrições médicas nas unidades de terapia intensiva de adulto doHospital Público da cidade de Vitória da Conquista/BA em 2017; - identificar o percentual de interações medicamentosas nas prescrições das UTI’s adultas do Hospital Geral de Vitória da Conquista; - verificar qual o tipo de interação mais frequente; - classificar as interações 
medicamentosas mais frequentes em relação a gravidade e, - Avaliar quais medicamentos mais prescritos que apresenta interação medicamentosa.

\section{Referencial Teórico}

\section{A Doença no Hospital Geral}

São diversas as dificuldades que os pacientes passam ao enfrentar e aceitar uma doença. Em virtude disso, vários sentimentos são despertados nesse momento.

De acordo com Couto apud Mota, Martins e Véras (2006) a internação é, em grande parte dos casos, algo que remete a sentimentos de insegurança, medo, angústia, etc. A situação de hospitalização deixa o paciente fragilizado, com sentimentos de solidão e abandono, além do medo de estar internado em uma UTI, levando em conta que é o ambiente em que os deixa mais distante da família e amigos e mais dependentes da equipe de profissionais que os cercam.

Nessa situação, requer-se um cuidado maior, já que as pessoas estão e psicologicamente dependendo principalmente dos profissionais da unidade, levando em conta também que esses pacientes estão em grande parte de seu tempo de internação afastados de familiares e amigos.

Por isso, entende-se que a internação hospitalar se posiciona como uma forma de confirmação da patologia, pois é um ambiente no qual o paciente se sente vulnerável e totalmente dependente e a mercê dos cuidados da equipe do hospital.

Para Jeammet e Consoliapud Mota, Martins e Véras (2006), a hospitalização apresenta, por si mesma, condições suficientes para induzir um efeito de estresse e de desorganização do controle emocional do paciente. Existe uma angústia muito grandepor não saber a natureza de sua doença, acrescendo-se dos efeitos físicos vivenciados a partir da doença.

De acordo com Couto apud Mota, Martins e Véras (2006), os pacientes de hospitais públicos têm sobre estes uma imagem já previamente formada. Vendo como estabelecimentos onde faltam médicos e o atendimento em grande parte dos casos é ruim, sendo que, qualquer tipo de cuidado dado a este paciente é muito baixo ou inexistente. Nesse contexto, vale 
ressaltar que na maioria das vezes a relação que se estabelece, entre médico e paciente é de subordinação e não de uma relação pautada em um cuidado da equipe multidisciplinar.

Quando um paciente precisa dos cuidados da Unidade de Terapia Intensiva, pelo fator cultural, já se sente amedrontado, por todo o contexto que essa unidade representa para a sociedade de uma maneira geral.

Então, os profissionais da saúde precisam atuar como uma equipe bem orientada para saber lidar com os sentimentos do paciente de uma forma sensível. A doença nunca será tarefa fácil para se trabalhar, no entanto é preciso que se faça o possível para propiciar um ambiente visando o cuidado do paciente.

\section{Unidade de Terapia Intensiva - UTI}

Durante o início do século XX, quando ocorria alguma complicação das cirurgias, a equipe de profissionais responsáveis oferecia o tratamento adequado para a recuperação do paciente naquele momento. Entendendo que esses fatos ocorriam com frequência, surgiram os centros de recuperação para recém-operados, que lá permaneciam até que atingissem uma condição clínica estável, sendo estes, os precursores dos futuros centros de terapia intensiva. (YUNES et al, 2011).

Então, de acordo com Yunes et al (2011), a partir de 1950, foram criadas as primeiras Unidades de Terapia Intensiva (UTI), hoje expandidas no mundo todo e reconhecidas pelos médicos, e entendida pela população leiga como uma unidade hospitalar onde são utilizados técnicas e procedimentos médicos, que podem propiciar condições para a reversão das enfermidades que colocam em risco a vida do paciente.

Em nosso país as primeiras UTIs foram criadas entre o final da década de 60 e o início dos anos 70 a partir dos centros de recuperação dos hospitais universitários e desenvolvidas principalmente dentro dos hospitais privados. Portanto, a UTI é um local que possui equipamentos, materiais e profissionais especializados para oferecer uma assistência adequada e o tratamento aos pacientes. (YUNES et al, 2011).

Os serviços que ocorrem na Unidade de Terapia Intensiva, na visão de Leite e Vila (2005), são destinados ao atendimento de pacientes críticos que necessitem de cuidados complexos e especializados. Esses serviços têm como objetivos: concentrar recursos humanos 
e materiais para o atendimento de pacientes graves que exigem assistência permanente, além da utilização de recursos tecnológicos apropriados para a observação e monitorização contínua das condições vitais do paciente e para a intervenção em situações de descompensações.

Sabe-se que existe uma probabilidade muito alta de grande parte dos problemas hospitalares estarem relacionados à medicação. Esse sistema de medicação vai desde a prescrição e distribuição até a administração do medicamento ao paciente, então oferecer qualidade de todas as etapas desse sistema depende de uma equipe de profissionais, podendo ocorrer erros de medicação em qualquer uma das etapas. (YUNES et al, 2011).

A partir disso, entende-se que a Unidade de Terapia Intensiva é um setor hospitalar de serviço especializado, sendo que, existe uma grande gama de medicamentos prescritos nessa unidade e, consequentemente um alto índice de interações medicamentosas. Essa situação será abordada no tópico seguinte.

\section{A Importância dos medicamentos no Âmbito Hospitalar}

Sem dúvidas os fármacos surgiram para proporcionar muitos benefícios a quem precisa deles, sendo um dos determinantes para a recuperação da saúde de um doente ou no auxilio e combate a doenças.

No entanto, também é preciso considerar os malefícios que o uso indevido de fármacos pode trazer a saúde.

Nesse sentido, Melo et al. (2006) elucida que se reconhece a necessidade constante de uma formulação e aplicação de políticas a favor de medicamentos essenciais e que promovam o seu uso racional, considerando que existem muitos problemas referentes a prescrição e aos problemas relacionados à falta de informação confiável sobre medicamentos.

Ainda no começo do século XIX eram utilizados na maioria dos casos medicamentos, estes eram remédios de origem natural, de estrutura química e natureza desconhecida. (LAPORTE, TOGNONI, ROSENFELD apud MELO; RIBEIRO; STORPIRTIS, 2006).

Após 1940,ocorreu a entradaem massa de novos medicamentos, que puderam trazer à população a possibilidade de cura para enfermidades que antes eram vistas como fatais, principalmente se tratando de doenças infecciosas. Os avanços nas pesquisas de novos

6 Id on Line Rev. Mult. Psic. V.11, N. 39., 2017 - ISSN 1981-1179 Edição eletrônica em http://idonline.emnuvens.com.br/id 
fármacos, em conjunto com a sua promoção comercial, criaram uma grande crença da sociedade em relação aos efeitos milagrosos dos medicamentos. (LAPORTE, TOGNONI, ROSENFELD apud MELO; RIBEIRO; STORPIRTIS, 2006).

No âmbito hospitalar,o fármaco então pode atuar no combate as enfermidades, trazendo seus efeitos benéficos, contribuindo para a recuperação do paciente.

Contrapondo essa ideia de que o medicamento é um mero complemento Nascimento apud Melo, Ribeiro e Storpirtis (2006)afirmam que a produção de medicamentos em escala industrial, fez com que eles alcançassem papel central na terapêutica. Sendo assim, a prescrição medica dos fármacos torna-se praticamente obrigatória nas consultas médicas.

Nessa visão, os fármacos, além de serem os principais aliados no tratamento, eles desempenham um papel onde a sua prescrição é crucial para a melhora do paciente.

No entanto, acredita-se que a grande importância do medicamento está relacionada ao seu valor simbólico e a capacidade de modificar o curso "natural" da doença que está sendo tratada. Então, prescrevê-lo passou a ser o resultado final de um processo de diagnóstico e decisão, em que os fármacos são, na maioria das vezes, o resumo da atitude e das esperanças do médico em relação ao curso de uma doença. (LAPORTE; TOGNONI; ROSENFELD apud MELO; RIBEIRO; STORPIRTIS, 2006).

Atualmente, o fato de o medicamento ter se tornado uma ferramenta tão familiar aos médicos aumenta o risco de sua utilização irracional. A própria prescrição reflete-se na disponibilidade de fármacos, a informação que foi distribuída sobre eles, que chegou ao médico e que ele interpreta. Desse conhecimento sobre o efeito das informações sobre o medicamento na hora da prescrição, nasce a preocupação com sua quantidade e qualidade.(MELOet al, 2006).

Com isso, o fácil acesso aos medicamentos pode fazer com que o seu uso se torne irracional e abusiva e, a consequência disso, é o excesso de medicalização, onde se preza mais pela quantidade.

O fácil acesso ao medicamento, a falta de informação sobre o assunto e a promoção farmacêutica distorcida e desenfreada leva a vários problemas, dentre eles está a escolha inadequada de medicamentos, exposições indevidas a reações adversas que podem ser tóxicas ou fatais, o aumento da resistência bacteriana, oaumento da automedicação, bem como os seus riscos e o desperdício de dinheiro. (CASTRO apud MELO; RIBEIRO; STORPIRTIS, 2006).

7 Id on Line Rev. Mult. Psic. V.11, N. 39., 2017 - ISSN 1981-1179 Edição eletrônica em http://idonline.emnuvens.com.br/id 
Melo et al (2006) cita que a medicação assumiu um papel importante culturalmente e cientificamente no tratamento da doença. No entanto, torna-se imprescindível que haja um conhecimento sobre o fármaco utilizado, resultando em seu uso consciente, para que esse possa de forma efetiva auxiliar no combate a enfermidade.

A partir disso, percebe-se a importância do medicamento no combate as enfermidades, porém, esse precisa ser sempre bem analisado e a equipe precisa realmente conhecer aquele fármaco para que possa auxiliar o paciente da melhor forma possível.

\section{Interação Medicamentosa}

Para Silva e Santos (2010), o medicamento, como recurso terapêutico, pode apresentar vários aspectos, como a descoberta de novas substâncias com melhores características permite o combate de doenças. Por outro lado, o seu emprego inadequado pode provocar eventos adversos diminuindo a efetividade dos tratamentos medicamentosos já existentes.

Segundo Hoefler (2005) existem três classificações para as interações medicamentosas, onde ele cita as interações farmacocinéticas, as quais, um fármaco altera a velocidade ou a extensão de absorção de outro fármaco. As interações de efeito, que ocorrem quando dois ou mais fármacos em uso concomitante têm ações farmacológicas similares ou opostas. E por fim, as interações farmacêuticas, que ocorrem antes da administração dos fármacos no organismo, quando se misturam dois ou mais deles em um mesmo recipiente.

A interação medicamentosa, então, ocorre quando os efeitos de um fármaco são alterados pela presença de outro e os resultados disso podem ser tanto positivos, como o aumento da sua eficácia, como negativos, havendo a diminuição da eficácia ou toxicidade. (HAMMES; PFUETZENREITER; SILVEIRA; KOENIG; WESTPHAL, 2008).

Ainda sobre o efeito nocivo que uma interação medicamentosa pode causar no organismo humano, Hoefler (2005) salienta acerca da redução da eficácia do fármaco, o que em alguns casos, pode também levar o paciente a morte.

No contexto hospitalar, Silva e Santos (2010) abordam que, ao longo dos últimos anos tem-se evidenciado a presença de fatores que geram os erros no uso dos fármacos o que 
consequentemente diminui a segurança no tratamento e causa prejuízos que vão da ocorrência de eventos adversos até a morte.

Dentre os casos de interação medicamentosa cabe também destacar as que ocorrem de forma benéfica, pois se tratam de uma interação desejada, de forma prescrita pelo médico ou profissional responsável. (HOEFLER, 2005).

Hoefler (2005) cita como exemplo o uso de varfarina que pode causar sangramentos se houver interação com um antiinflamatório não esteroide (AINE) sem reduzir a dose do anticoagulante. Outro exemplo dizendo que a tetraciclina sofre quelação por antiácidos e alimentos lácteos, sendo excretada nas fezes, sem produzir o efeito antimicrobiano desejado.

Portanto, entende-se que em um âmbito geral a interação medicamentosa pode ser um risco a saúde como também um auxilio para a medicina e pacientese, a partir disso, percebe-se a importância estudar acerca da prescrição dos medicamentos, já que isso pode causar diversos tipos de efeitos para o organismo humano.

Por isso, no ambiente das unidades de Terapia Intensiva, o qual é o foto desse trabalho, precisa-se pensar mais sobre essa situação.

Um estudo realizado dentro da UTI adulto de um Hospital privado de Minas Gerais aponta que a incidência das interações medicamentosas pode variar de 3\% a 5\% nos pacientes que recebem poucos medicamentos e de até $20 \%$ nos pacientes que recebem de 10 a 20 drogas. Entendendo que a frequência de interações em pacientes em estado critico de saúde pode ser alta, visto o grande número de drogas que recebem. Esse estudo evidenciou alta prevalência de interações na UTI, principalmente do tipo moderada, levando-se em conta as condições clínicas desfavoráveis do paciente, alterações fisiológicas e em muitos casos a idade avançada, esse tipo de interação acabam por se tornar mais relevantes. (YUNES; COELHO; ALMEIDA, 2011).

Um outro estudo destaca que os pacientes em UTI tem alta prevalência de interações medicamentosas potenciais, sendo que o número de fármacos por dia é fator de risco independente para aumento desta possibilidade. Mas, por outro lado entende-se que a maior parte das interações medicamentosas potenciais não é uma contraindicação para a utilização do fármaco, não sendo necessário substitui-lo ou suspender seu uso, o autor ainda afirma que o reconhecimento das interações pode ocorrer na prescrição, dispensação e administração das medicações. (HAMMES; PFUETZENREITER; SILVEIRA; KOENIG; WESTPHAL, 2008). 
O uso da terapia a partir de medicamentos na unidade de terapia intensiva (UTI) está relacionada às atividades mais desenvolvidas pelos profissionais do setor, aos quais cabe a responsabilidade pelo aprazamento, preparo, administração, monitoramento da medicação e avaliação do paciente quanto a possíveis complicações. (SILVA; SANTOS, 2010).

Sobre isso, na pesquisa de Gimenes, Baroni e Rodrigues (2014), eles chegaram as seguintes conclusões sobre os dados coletados:

\begin{abstract}
O elevado número de IMP em prescrições da UTI adulto, sendo 92,57\% das IMP classificadas quanto a severidade em grave ou moderada. Ressalta a necessidade da presença do farmacêutico clínico nessa área, visando identificar as possíveis interações medicamentosas, ampliando o conhecimento sobre os riscos, benefícios e manejo clínico das mesmas, prevenindo o surgimento de eventos adversos a medicamentos, diminuindo o custo e o tempo de internação, aumentando assim, a qualidade e a segurança da assistência prestada aos pacientes internados. (p.23).
\end{abstract}

Entendendo isso, faz-se necessária a busca, por parte dos profissionais que trabalham tanto no âmbito hospitalar, quanto clinico, da garantia de uma prática medicamentosa segura, em que possíveis interações medicamentosas possam ser previstas e impedidas. (SILVA; SANTOS, 2010).

Os profissionais inseridos nesse ambiente precisam buscar e se conhecer melhor sobre as interações medicamentosas e seus possíveis riscos para os pacientes para realizar uma prática mais consciente.

Sabendo da grande expansão de novos fármacos, entende-se também que a ocorrências de prescrições mais complexas com relação a suas combinações ocorram, assim sendo, se torna extremamente difícil para médicos, farmacêuticos ou profissionais responsáveis reconhecerem potenciais interações. (HAMMES; PFUETZENREITER; SILVEIRA; KOENIG; WESTPHAL, 2008).

Em relação as prescrições, existe algumas que são mais difíceis de serem identificadas as possíveis interações. No entanto, os profissionais também necessitam levar em consideração alguns fatores particulares dos pacientes.

Existem alguns fatores de risco que predispõem a ocorrência de interações medicamentosas, sendo que,a gravidade dessas interações dependem de alguns fatores, como o número de medicações prescritas, duração do tratamento, idade do paciente e estados de doença. (HAMMES; PFUETZENREITER; SILVEIRA; KOENIG; WESTPHAL, 2008). 
Nesse contexto, consequentemente, pacientes que requerem grande número de fármacos com um longo tempo de tratamento e que apresentem alterações orgânicas relacionas a idade, ou doenças como insuficiência renal, hepatopatias como a cirrose e hepatites virais agudas são considerados de alto risco para interações medicamentosas severas. (HAMMES, et al, 2008).

Por isso, existem alguns casos nos quais os pacientes já tomam diversos medicamentos e, nesses casos, precisa-se ter um cuidado redobrado, pois, como visto, eles correm um risco maior de apresentar interações medicamentosas negativas.

Segundo um estudo realizado pela Harvard Medical PracticeStudy II, aponta que, as medicações e suas complicações são o tipo de caso adverso mais comum na internação hospitalar, ocorrendo em 19\% dos pacientes, sendo que, 2-3\% desses pacientes hospitalizados experimentam reações provocadas especificamente por interações farmacológicas.(HAMMES et al, 2008).

Estudos revelam que em unidades de terapia intensiva (UTI), interações medicamentosas possam ocorrer em $44.3-95 \%$ dos pacientes. No entanto, essas pesquisas os são insuficientes e limitadas em relação a medida do real valor clínico destas. (HAMMES et al, 2008).

Em um estudo baseado em prescrições médicas em três UTI de Joinville-SC, que buscou constatar a prevalência de interações medicamentosas potenciais (IMP), classificar seu valor clínico e identificar possíveis fatores de risco.Encontrou-se que, a classe farmacológica que teve maior prevalência foram os antimicrobianos apresentando $23 \%$. A segunda classe mais prevalente foi a dos anticonvulsivantes, com 10,2\%, os anti-hipertensivos também com 10,2\%. (HAMMES et al, 2008).

$\mathrm{Na}$ pesquisa de Hammeset al (2008), os pacientes do grupo com Interação Medicamentosa Potencial utilizaram maior número de fármacos durante a internação, maior número de medicamentos por dia e maior tempo de internação na UTI. Com isso, orisco de aumento de ocorrências de IMP significativas foi o número de uso de fármacos por dia, sendo que o risco aumenta quando mais de seis variedades medicamentosas são utilizadas. (HAMMES et al, 2008).

No estudo de Alvim et al (2015), foi abordado sobre a classe que mais se destaca nas prescrições das UTI: 
Verifica-se que os antimicrobianos são uma classe cuja prescrição é frequente na unidade de terapia intensiva, apresentando elevada quantidade de interações medicamentosas potenciais, sendo a maior parte das interações medicamentosas potenciais considerada altamente significativa. (p. 358).

Então, entende-se que ofato de interações adversas ocorrerem entre medicamentos utilizados ocorre de maneira proporcional à quantidade de fármacos usados simultaneamente, sendo que, a frequência de interações medicamentosas significativas, saltam de $3 \%$, em pacientes com uso de até seis medicamentos para $20 \%$ quando é feito uso de dez medicamentos diferentes. Compreendendo que nas UTIs os pacientes fazem uso de muitos medicamentos, e consequentemente existe o aumento em quase três vezes o risco de interação. (SILVA; SANTOS, 2010).

Contudo, as interações medicamentosas são percebidas como resposta farmacológica ou clínica, obtida através do uso de medicamentos, o que ocasiona efeitos diferentes daqueles que ocorrem quando os medicamentos são dados individualmente. $\mathrm{O}$ resultado final pode aumentar ou diminuir os efeitos desejados ou causar efeitos adversos. (SILVA; SANTOS, 2010).

As interações graves são aquelas que colocam a vida do paciente em risco ou que possam causar danos permanentes. As interações moderadas são aquelas nas quais os efeitos causam alguma alteração clínica no paciente exigindo tratamento adicional, hospitalização ou aumento no período de internação. E por fim, as leves dizem respeito a efeitos mais suaves, podendo ser incômodos ou passarem despercebidos, sem afetar significativamente o efeito da terapia. (SILVA; SANTOS, 2010).

Portanto, o uso de fármacos deve ocorrer de forma consciente, com a prescrição racional por uso do médico ou profissional responsável e pelo uso coerente do paciente.

Além disso, percebeu-se que as interações medicamentosas precisam preferencialmente ocorrer de forma prevista e analisada pelo médico, ao contrário, pode trazer riscos a vida do paciente, principalmente se tratando de um usuário da Unidade de Terapia Intensiva, onde o paciente se encontra ainda mais frágil, devido a condições emocionais e físicas.

Além disso, o medicamento precisa ser visto como auxiliar no tratamento de uma doença e não o condutor deste tratamento é preciso que haja informação sobre os fármacos para que com isso seu aproveitamento terapêutico seja eficaz. 
No estudo de Faroni (2016), foi possível observar isso, concomitante com algumas situações que podem potencializar a interação medicamentosa:

\begin{abstract}
A falta de informação do usuário sobre os medicamentos utilizados; e a necessidade do farmacêutico nas ações relacionadas ao uso do medicamento e acompanhamento farmacoterapêutico. Além disso, constatou-se que a maioria dos usuários tinha receio de perguntar ao médico sobre o tratamento, devido ao tempo escasso das consultas e o contato pouco humanizado conforme preconizado no SUS. Por conseguinte, é imprescindível promover estratégias que promovam a educação continuada sobre o tema, capacitando os profissionais para que possam informar, detectar e prevenir as potenciais interações medicamentosas, otimizando o tratamento e o cuidado integral dos usuários na perspectiva do Sistema Único de Saúde. (p. 17).
\end{abstract}

Nesse sentido, é necessário também promover estratégias de prevenção relacionadas as interações medicamentosas e isso pode ocorrer através de um diálogo aberto entre a equipe e os pacientes.

Através de um espaço mais humanizado, a equipe pode conscientizar os pacientes sobre os riscos dessas interações, fazendo com que estes também perguntem a respeito de suas dúvidas e angústias para a equipe.

Com isso, se torna imprescindível pesquisar sobre as interações medicamentosas que ocorrem em uma UTI adulta, para que se possa estuda-las e evita-las em casos de malefícios a vida do paciente.

\title{
Metodologia
}

\section{Tipo de Pesquisa}

O trabalho trata-se de uma pesquisa quantitativa de natureza analítica, onde, segundo Chizzotti (1998), a pesquisa quantitativa é aquela que prevê a mensuração das variáveis prédeterminadas, buscando verificar e explicar sua existência, relação ou influência sobre outra variável. Este tipo de pesquisa se utiliza da análise da frequência de ocorrência para medir a veracidade ou não daquilo que está sendo investigado.

Para Gil (1994), esse tipo de pesquisa trata-se de tudo pode ser quantificável, o que significa traduzir em números, opiniões e informações para classificá-los e analisá-los, requerendo o uso de recursos e técnicas estatísticas. 
Segundo Gil (2010), quando se faz uma pesquisa com uma metodologia quantitativa está se buscando estabelecer uma regra, um princípio, algo que reflita a uniformidade do fenômeno (ou parte dele), preocupando-se com o que é comum à maioria das situações.

A natureza analítica da pesquisa diz respeito ao envolvimento mais aprofundado das informações que são coletadas no estudo, observacional ou experimental. Tentando explicar o contexto de um fenômeno no âmbito de um grupo ou população. Sendo mais complexa do que a pesquisa descritiva, levando em conta que procura explicar a relação entre a causa e o efeito. Podendo-se afirmar que a maior diferença entre um estudo descritivo e um analítico é a capacidade deste último fazer predições para a população de onde a amostra foi retirada, e fazer inferências estatísticas pela aplicação de testes de hipótese.(FONTELLES; SIMÕES; FARIAS; FONTELLES, 2003).

O delineamento utilizado nesta pesquisa foi o estudo transversal,sendo assim conhecido, pois, pesquisa é realizada em um curto período de tempo e em um determinado momento. (FONTELLES et al, 2003).

Tratando-se de um estudo retrospectivo, é necessário citar que este é utilizado para explorar fatos do passado, podendo ser delineado para retornar, do momento atual até um determinado ponto no passado, há vários anos, etc. (FONTELLES; SIMÕES; FARIAS; FONTELLES, 2003).

A amostra a ser analisada foi composta pela segunda via das prescrições médicas das Unidades de Terapia Intensiva 1 e 2 de adulto entregues à farmácia, no período de 15 dias do decorrente ano.

Os critérios de inclusão foram pacientes com idade superior a 18 anos, que apresentem o tempo de permanência na Unidade de Terapia Intensiva de pelo menos 24 horas e que a prescrição medicamentosa contenha dois ou mais medicamentos. O critério de exclusão utilizado serão as prescrições repetidas.

\section{Local da Pesquisa}

A pesquisa foi realizada nas duas Unidades de Terapia Intensiva de adulto do Hospital Geral de Vitória da Conquista BA. 


\section{Instrumentos da Pesquisa}

As prescrições medicamentosas que fizeram parte da pesquisa serão analisadas através do banco de dados Micromedex Healthcare Series ${ }^{\circledR}$.

O manual do produto traz que esse programa é uma ferramenta desenvolvida para apoiar o trabalho clínico na atenção ao paciente. Com isso, ele pode proporcionar aos profissionais responsáveis, um acesso fácil à informação clínica, sendo ideal para os profissionais de saúde que desejam uma informação imparcial sustentada em revisões sistemáticas acerca de medicamentos, toxicologia, doenças, cuidado primário, educação do paciente e medicina alternativa. (HEALTHCARE, 2009).

\section{Análise dos Dados}

A técnica de análise dos dados coletados foi através da interpretação das informações coletadas nas prescrições médicas da UTI 1 e 2 de adulto entregues à farmácia, no período de 15 dias desse ano, articulando essas informações com as teorias existentes.

\section{Aspectos Éticos}

Antes da execução deste projeto, o mesmo passou por procedimentos éticos que se iniciam pela apresentação e avaliação do projeto para a instituição participante e os responsáveis pelas Unidades de Terapia Intensiva adulta do Hospital Geral de Vitória da Conquista BA. Após a concordância da instituição, o mesmo forneceu a assinatura da declaração para que se proceda com à pesquisa.

Depois, a pesquisa foi avaliada pelo Comitê de Ética em Pesquisa da FAINOR. 


\section{Riscos e Benefícios da Pesquisa}

Existe a possibilidade de um desconforto e risco mínimo aos participantes e/ou responsável, durante a coleta do material para a pesquisa devido à necessidade de expor sobre sua doença e o uso de medicamentos mesmo que de forma indireta.

Contudo, se justifica pelo fato de possibilitar que a frequência das interações medicamentosas sejam mensuradas e consequentemente haja uma analise por parte dos profissionais responsáveis sobre seus riscos e benefícios para a saúde do paciente.

\section{Resultados}

A amostra estudada na Unidade de Terapia Intensiva (UTI) compreendeu 204, pacientes, $135(66,2 \%)$ do sexo masculino, o qual apresentou uma maior percentual. A idade média dos pacientes é de 47,3 \pm 21 . O número de prescrição na UTI 1 é de 90 e na UTI 2 é de 114. O número de medicamentos foi de 2368 em 204 prescrições, com uma média de 11,6 2,98 .

A partir da análise dos dados por meio do Micromedex, houve 1049 registros, dentre eles 1036 foi de interações, sendo que 601 (57,29\%) foi de gravidade importante; 21 (2\%) de gravidade contraindicada; 368 (35,08\%) de gravidade moderada; $46(4,38 \%)$ de gravidade secundária e 13 (1,23\%) não houve interação. A média de interações por prescrição foi de \pm 5,7. O gráfico 1 apresenta a porcentagem das interações medicamentosas:

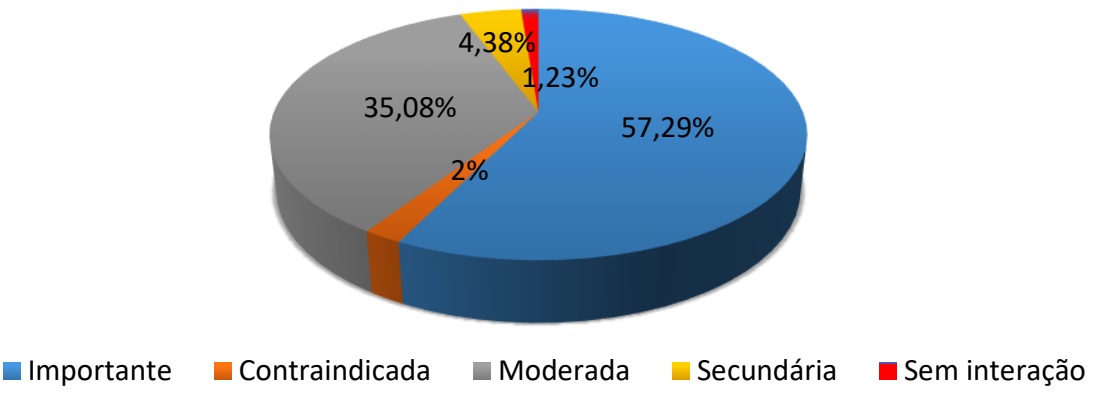

Gráfico 1. Porcentagem de potenciais de IM encontradas nas prescrições de pacientes internadas nas UTI’s. As IM foram classificadas em "importante", "contraindicada", "moderada" e "secundária" de acordo com a base de dados Micromedex Healthcare Series®. 
Com isso, é necessário trazer acerca da classificação de gravidade existente no banco de dados eletrônicos Micromedex Healthcare Series ${ }^{\circledR}$ e o que significa cada uma delas. O quadro a seguir, baseado nos autores Hutchison, Shahan e Anderson (2014, p. 13), traz essa classificação:

Quadro 1. Classificação de Interações Medicamentosas.

\begin{tabular}{|c|c|c|c|}
\hline Contraindicado & $\begin{array}{c}\text { Maior } \\
\text { (importante): }\end{array}$ & Moderado: & Menor (secundário) \\
\hline $\begin{array}{l}\text { O uso concomitante dos } \\
\text { medicamentos } \\
\text { contraindicado. }\end{array}$ & 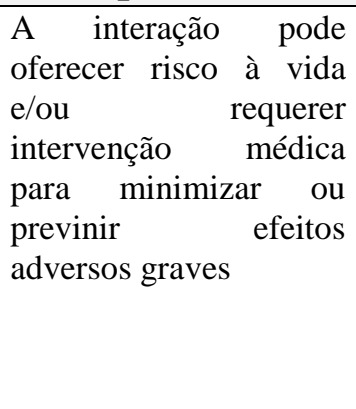 & $\begin{array}{l}\text { A interação pode } \\
\text { provocar exacerbação } \\
\text { da condição do paciente } \\
\text { e/ou requerer alteração } \\
\text { no tratamento. }\end{array}$ & $\begin{array}{l}\text { A interação teria efeitos } \\
\text { clínicos limitados. As } \\
\text { manifestações podem } \\
\text { incluir aumento na } \\
\text { frequência ou na } \\
\text { gravidade dos efeitos } \\
\text { colaterais, mas em geral } \\
\text { não seria necessária } \\
\text { qualquer alteração } \\
\text { importante no tratamento. }\end{array}$ \\
\hline
\end{tabular}

As interações medicamentosas classificadas como graves e como contraindicadas e, a sua frequência nas prescrições para os pacientes internados nas UTIs dessa pesquisa, descritas na tabela 1, foram:

Tabela 1. Interações medicamentosas classificadas como "contraindicadas" pela base de dados Micromedex Healthcare Series ${ }^{\circledR}$ por frequência de aparecimento nas prescrições de pacientes internadas na UTI 1 e 2.

\begin{tabular}{lll}
\hline Medicamento1 & Medicamento 2 & Frequência (\%) \\
\hline Haloperidol & Metoclopramida & $28,55 \%$ \\
Metoclopramida & Risperidona & $28,55 \%$ \\
Escitalopram & Metoclopramida & $19 \%$ \\
Fluconazol & Metadona & $14,30 \%$ \\
Clorpromazina & Metoclopramida & $4,80 \%$ \\
Gluconato de cálcio & Ceftriaxona & $4,80 \%$
\end{tabular}


2, foram:

De gravidade importante foram $601 \mathrm{e}$, as que mais recorrentes, de acordo com a tabela

Tabela 2. Interações medicamentosas classificadas como "importante" pela base de dados Micromedex Healthcare Series ${ }^{\circledR}$ por frequência de aparecimento nas prescrições de pacientes internadas na UTI 1 e 2.

\begin{tabular}{llc}
\hline Medicamento 1 & Medicamento 2 & Frequência (\%) \\
\hline Dipirona & Enoxaparina & $37 \%$ \\
Fentanil & Fenitoina & $13 \%$ \\
Fentanil & Midazolam & $13 \%$ \\
Metoclopramida & Tramadol & $10 \%$ \\
Diazepam & Fenitoina & $6 \%$ \\
Dipirona & Furosemida & $5 \%$ \\
Dipirona & Prednisona & $5 \%$ \\
Diazepam & Fentanil & $4 \%$ \\
Dipirona & & $4 \%$ \\
Fentanil & Hidroclorotiazida & $3 \%$ \\
\hline
\end{tabular}

Dentre os medicamentos mais prescritos que apresentaram interação medicamentosa de gravidade "importante" foram classificados de acordo com o Anatomical Therapeutic Chemical (ATC), os 15 medicamentos mais prescritos no período de 15 dias.

Tabela 3. Classificação ATC de medicamentos que apresentaram IM classificada como "importante" através da base de dados Micromedex®.

\begin{tabular}{lcc}
\hline MEDICAMENTO & \multicolumn{2}{c}{ CLASSIFICAÇÃO ATC } \\
\hline dipirona & Sem classificação ATC \\
fentanil & N02A & Opiáceos \\
enoxaparina & B01A & Antitrombolíticos \\
fenitoina & N03A & Antiepilépticos \\
midazolam & N05C & Hipnóticos e Sedativos \\
diazepam & N05B & Ansiolítico \\
tramadol & N02AX & Outros opióides
\end{tabular}


metadona

opióides

metoclopramida

haloperidol

fenobarbital

morfina

furosemida

prednisona

N07BC Medicamentos utilizados na dependência de

A04A

N05A

N03A

N02A

$\mathrm{C} 03 \mathrm{C}$

$\mathrm{H} 02 \mathrm{~A}$
Antieméticos e antinauseantes

Antipsicóticos

Antiepilépticos

Opiáceos

Diuréticos de alça

Corticosteróides

\title{
Discussão
}

Nesse estudo foi possível visualizar o uso e a prescrição de medicamentos. Com base nisso, percebe-se que o número de interações foi de 1036, sendo que 601 (57,29\%) foi de gravidade importante e, isso nos faz pensar os possíveis riscos que isso pode causar devido a falta de critérios por parte de alguns profissionais a respeito da compreensão das interações medicamentosas.

A interação medicamentosa de gravidade importante que mais ocorreu foi entre dipirona e enoxaparina, com 134 interações. Além disso, entre todos, o dipirona foi o medicamento que mais interagiu com outros. Sobre a dipirona:

\begin{abstract}
A importância da dipirona venosa como fármaco analgésico nos processos álgicos de curta duração $(<5)$ decorre de sua alta eficácia no tratamento de dores agudas fracas e medianas, seu baixo custo, ampla e ubíqua disponibilidade, boa margem de segurança, mormente nos pacientes hepatopatas, nefropatas e cardiopatas; possibilidade de associação com outros analgésicos: analgesia pré-emptiva e multimoda Sua prescrição ou não do arsenal terapêutico dependerá de futuro estudo multicêntrico internacional sob a luz da medicina baseada em evidência (...) Em função da dose, a dipirona comporta-se como fármaco antitérmico, analgésico e, potencialmente, antiinflamatório, sendo referendado diuturnamente pelo seu amplo uso como agente principal ou coadjuvante no tratamento das dores agudas na SRPA, UTI, enfermaria e clínicas odontológicas. (VALE, 2006 p.119).
\end{abstract}

Compreende-se que o alto índice de uso para esse medicamento nas UTIs estudadas decorre pelo fato de que ele é prescrito, por exemplo, para pacientes com dores devido a acidentes e em casos de pacientes cardiopatas. No entanto, é necessário um cuidado para a prescrição da dipirona, já que ele apresenta diversas interações de gravidade importante. 
Outro medicamento que apresentou interações maiores e, teve um uso elevado, foi o fentanil, que, "é um opioide de ação rápida e eficiente, cem vezes mais potente que a morfina. Além de seguro, potencializa o efeito sedativo dos benzodiazepínicos, diminuindo a necessidade de doses maiores desses agentes". (LIRA-FILHO, et al p. 84).

Nesse sentido, percebe-se que é indispensável abordar sobre a relevância de compreender os medicamentos, principalmente os que oferecem mais riscos, pois, eles podem ser bastante prejudiciais aos pacientes internados nas UTIs.

É necessário estudar sobre a interação dos prováveis medicamentos que o paciente irá utilizar, no intuito de adquirir conhecimento sobre os riscos, benefícios e manejo clínico, prevenindo o surgimento de eventos adversos aos medicamentos, como o desenvolvimento de condições que afetam a saúde do paciente e que, inclusive podem levar a morte se a interação for de risco maior. Assim, a interação entre medicamentos deve ser muito bem analisada antes da prescrição. (MAZZOLA, RODRIGUES, 2011).

Vários são os efeitos de uma prescrição que não abranja uma análise envolvendo os riscos-benefícios do uso de medicamentos de uma maneira concomitante. Para se precaver de possíveis complicações, torna-se crucial um olhar para essas questões.

A interação entre medicamentos precisa ser avaliada, pois, podem resultar em reações perigosas. Por isso, tem-se a necessidade da atuação de uma equipe multidisciplinar que analise cada caso em especifico, compreendendo o lado positivo e lado negativo interações, principalmente as de gravidade importante e contraindicada. Cabe ainda ressaltar que, um uso irracional que desconsidere isso, pode levar a internação de pacientes por um período maior, prejudicando o paciente e elevando o custo gasto pelo hospital. (MAZZOLA, 2016).

Assim, antes da prescrição de mais de um medicamento, é fundamental que a equipe multidisciplinar avalie os possíveis riscos dessa interação, para que, não prejudique a saúde do paciente, devido ao fato de que a interação maior pode levar até a morte. Por isso, deve-se prestar atenção ao uso desenfreado de medicamentos.

Os cuidados em saúde são muito mais do que uma intervenção mecanicista e breve. Compreender a totalidade do sujeito e as possíveis implicações globais do medicamento para ele é uma atitude que todo o profissional da saúde deve ter.

Para suscitar possíveis soluções para isso, a qualidade de assistência prestada aos pacientes internados é fomentada por meio de discussões dos protocolos, através do estudo dos medicamentos, reuniões de equipe e, por meio de visitas ao leito. Através disso, pode-se 
facilitar um maior conhecimento de como o paciente está reagindo as interações entre medicamentos. (MAZZOLA et al, 2011).

Mazzola (2016) também traz algumas estratégias para um controle mais severo, como a elaboração e a protocolarão de uma base de dados, com o intuito de promover o uso racional e controlado de medicamentos, com um número menor de complicações decorrentes de interações medicamentosas, mais segura para o paciente.

Diversas são as propostas que tem com o intuito de amenizar os riscos aos pacientes, assim sendo, propiciar uma base monitorada é decisivo para que ocorra menos problemas em relação as interações medicamentosas, promovendo uma internação mais segura, focando nas necessidades do paciente.

Por isso, é imprescindível pensar sobre alternativas, como o monitoramento, para

diminuir os possíveis riscos das interações e, concomitantemente a isso, com o monitoramento, os profissionais poderão avaliar se realmente vale a pena o uso de mais de um medicamento para o paciente em específico.

Além disso, o desenvolvimento de estratégias de ação que abordem sobre a educação continuada nessa temática, capacitando os profissionais da saúde, para que esses tenham mais recursos teóricos para compreender como ocorre as interações medicamentosas. Por meio disso, otimiza-se o tratamento e o cuidado integral dos pacientes. (FARAONI, 2016).

Nesse sentido, a educação continuada como meio de potencializar a aprendizagem dos profissionais, pode ser também uma das saídas para diminuir o índice da prescrição medicamentosa desenfreada, já que, em muitos casos, não se tem uma real necessidade de receitar tantos medicamentos.

\section{Conclusão}

Com esse estudo, foi possível considerar a importância dos profissionais das UTIs prestarem atenção nas interações medicamentosas, principalmente as de grau maior, já que, estas podem trazer diversas complicações aos pacientes.

A frequência de potenciais interações medicamentosas em prescrições médicas nas UTI’s adultas Hospital Público da cidade de Vitória da Conquista/BA no decorrente do ano de 
2017, apresentou um número bastante elevado, devendo ser suscitado debates com os profissionais para abarcar essa temática.

Por meio da amostra estudada na Unidade de Terapia Intensiva, percebeu-se o alto índice das interações medicamentosas, suscitando o debate para uma possível falta de critério na prescrição ou, inclusive, de uma falta de informação em termos de formação profissional para olhar a essas questões tão importantes.

Como estratégias de ação para a diminuição do uso irracional de medicamentos, podese fomentar discussão entre profissionais de diferentes áreas, reuniões de equipe, elaboração de protocolos de controle de medicamentos e visita aos leitos. Através disso, tem-se uma prescrição mais segura, com menos riscos aos pacientes.

Para futuras pesquisas, sugere-se analisar mais profundamente, interações mais especificas, por meio de um estudo de caso. Assim, será possível tecer considerações mais explicitadas acerca dos efeitos que estas acarretam para os sujeitos da pesquisa.

\section{Referências}

ANATOMICAL THERAPEUTIC CHEMICAL CLASSIFICATION SYSTEM. Disponível em: <https://www.whocc.no/atc_ddd_index/>. Acesso em: 25 de agosto de 2017.

ALVIM, Mariana Macedo et al. Eventos adversos por interações medicamentosas potenciais em unidade de terapia intensiva de um hospital de ensino. RevBras Ter Intensiva, v. 27, n. 4, p. 353-359, 2015.

CHIZZOTTI, Antônio. Pesquisa em Ciências Humanas e Sociais. 3.ed. São Paulo: Cortez, 1998.

FONTELLES, Mauro José; SIMÕES, Marilda Garcia; FARIAS, Samantha Hasegawa; FONTELLES, Renata Garcia Simões. Metodologia da pesquisa científica: diretrizes para a elaboração de um protocolo de pesquisa. Disponível em: <https://cienciassaude.medicina.ufg.br/up/150/o/Anexo_C8_NONAME.pdf>. Acesso em: 20 de maio de 2017.

GIL, Antonio Carlos. Como Elaborar Projetos de Pesquisa. 5. ed. São Paulo: Atlas S.A, 2010.

GIL, Antonio Carlos. Métodos e Técnicas de Pesquisa Social. 4. ed. São. Paulo: Atlas, 1994; 2007; 
GIMENES, A. H. S; BARONI, M. M. F.; RODRIGUES, P. J. N. Interações Medicamentosas Potenciais em unidade de terapia Intensiva adulto de um Hospital Público estadual. Rev. Bras. Farm. Hosp. Serv. Saúde São Paulo, v. 5, n. 4, p. 19-24, 2014.

HAMMES, Jean André Hammes; PFUETZENREITER, Felipe; SILVEIRA, Fabrízio da; KOENIG, Álvaro; WESTPHAL, Glauco Adrieno. Prevalência De Potenciais Interações Medicamentosas Droga-Droga Em Unidades De Terapia Intensiva. Disponível em: <http://www.scielo.br/pdf/rbti/v20n4/v20n4a06>. Acesso em: 20 de maio de 2017.

HEALTHCARE, Thomson.Manual Português Micromedex Healthcare Series.Disponível em:

〈https://www.periodicos.capes.gov.br/images/documents/Micromedex_Manual_2009.pdf>.

Acesso em: 20 de maio de 2017.

HOEFLER, Rogério. Estabilidade dos medicamentos após abertura. Revista Pharmacia Brasileira, Junho/Julho, nº 48, p. 49-51, 2005.

FARAONI, A. S. Possíveis interações medicamentosas entre usuários de uma unidade básica de saúde (ubs) do município de são cristóvão-SE. Saúde. com, v. 11, n. 1, 10-11, 2016.

LEITE, Maria; VILA, Abadia Vanessa da Silva Carvalho. Dificuldades Vivenciadas Pela Equipe Multiprofissional Na Unidade De Terapia Intensiva.Rev Latino-am Enfermagem, 13(2):145-50, 2005 março-abril;. Disponível em: <http://www.scielo.br/scielo.php?script=sci_arttext\&pid=S0104-11692005000200003>. Acesso em: 18 de maio de 2017.

MAZZOLA, Priscila Gava; RODRIGUES, Aline Teotonio; DA CRUZ, Aline Aparecida. Perfil e manejo de interações medicamentosas potenciais teóricas em prescrições de UTI. CEP, v. 13083, p. 887, 2011.

MELO, Daniela Oliveira de; RIBEIRO, Eliane; STORPIRTIS, Sílvia. A Importância E A História Dos Estudos De Utilização De Medicamentos. Revista Brasileira de Ciências Farmacêuticas BrazilianJournalofPharmaceuticalSciences vol. 42, n. 4, out./dez, 2006. Disponível em: <http://www.scielo.br/pdf/\%0D/rbcf/v42n4/a02v42n4.pdf>. Acesso em: 19 de maio de 2017.

MONTEIRO, Ruth Macêdo Monteiro; BARROSO, Maria Grasiela Teixeira. A Família e o Doente Mental Usuário do Hospital-Dia - Estudo De Um Caso. Rev.latinoam.enfermagem, Ribeirão Preto, v. 8, n. 6, p. 20-26, dezembro 2000. Disponível em: <http://www.revistas.usp.br/reeusp/article/view/41328/44897>. Acesso em: 20 de maio de 2017.

MOTA, Roberta Araújo; MARTINS, Cileide Guedes de Melo; VÉRAS, Renata Meira. Papel Dos Profissionais De Saúde Na Política De Humanização Hospitalar. Psicologia em Estudo, Maringá, v. 11, n. 2, p. 323-330, mai./ago. 2006. Disponível em: <http://www.scielo.br/pdf/\%0D/pe/v11n2/v11n2a10.pdf>. Acesso em: 19 de maio de 2017. 
SILVA, Lolita Dopicoda; SANTOS, Manassés Moura dos. Interações Medicamentosas Em Unidade De Terapia Intensiva: Uma Revisão Que Fundamenta O Cuidado Do Enfermeiro. Rev. enferm. 19(1):134-9, UERJ, Rio de Janeiro, 2011. Disponível em: <http://www.facenf.uerj.br/v19n1/v19n1a22.pdf>. Acesso em:.20 de maio de 2017.

YUNES, Luciana Palis; COELHO, Tamara de Almeida; ALMEIDA, Silvana Maria de. Principais Interações Medicamentosas Em Pacientes Da Uti-Adulto De Um Hospital Privado De Minas Gerais. Disponível em: $<$ http://www.sbrafh.org.br/rbfhss/public/artigos/RBFHSSV2N3\%20artigo04.pdf $>$. Acesso em: 21 de maio de 2017.

MAZZOLA, P. G. Avaliação de potenciais interações medicamentosas em prescrições de pacientes internadas, em hospital público universitário especializado em saúde da mulher, em Campinas-SP. Revista de Ciências Farmacêuticas Básica e Aplicada, v. 31, n. 2, p. 171$176,2010$.

MAZZOLA, P. G.; RODRIGUES, A. T.; DA CRUZ, A. A. Perfil e manejo de interações medicamentosas potenciais teóricas em prescrições de UTI. CEP, v. 13083, p. 887, 2011.

HUTCHISON T. A; SHAHAN P. R.; ANDERSON ML. Drug-Dex® System. Micromedex, Inc. Englewood, Colorado, 2014.

FARAONI, A. S. possíveis interações medicamentosas entre usuários de uma unidade básica de saúde (ubs) do município de São Cristóvão-se. Saúde. com, v. 11, n. 1, 2016.

LIRA-FILHO, E. B. et al. Impacto do fentanil associado ao midazolam na sedação para ecocardiogramatransesofágico. ABC., imagem cardiovasc, v. 27, n. 2, p. 83-86, 2014.

VALE, N. Desmistificando o uso da dipirona. Cavalcanti IL, Cantinho FA, Assad A. Medicina perioperatória. Rio de Janeiro: Editora da Sociedade de Anestesiologia do Estado do Rio de, p. 1107-24, 2006.

\section{Como citar este artigo (Formato ABNT):}

SILVA, Júlia S e; DAMASCENA, Rodrigo S. Avaliação das interações medicamentosas potenciais no âmbito da UTI adulta. Id on Line Revista ultidisciplinar e de Psicologia, 2017, vol.12, n.39, p.124. ISSN: 1981-1179.

Recebido: 13.11.2017

Aceito: 14.11.2017 